\title{
Stage IIIA Rectal Neuroendocrine Tumor AJCC v8
}

National Cancer Institute

\section{Source}

National Cancer Institute. Stage IIIA Rectal Neuroendocrine Tumor A/CC v8. NCI

Thesaurus. Code C135531.

Stage IIIA includes: T4, N0, M0. T4: Tumor invading the visceral peritoneum (serosa) or other organs or adjacent structures. N0: No regional lymph node metastasis. M0: No distant metastasis. (AJCC 8th ed.) 